\title{
Study of Magnetization Reversal Process in FeCo/Ru/FeCo Exchange Coupled Synthetic Antiferromagnetic Multilayers
}

\author{
Xi Liu, ${ }^{1}$ Shunji Ishio, ${ }^{2}$ and Hailin $\mathrm{Ma}^{1}$ \\ ${ }^{1}$ Key Laboratory of Ministry of Education on Opto-Electronic Technology and Intelligent Control, Lanzhou Jiaotong University, \\ LanZhou, Gansu 730070, China \\ ${ }^{2}$ Department of Materials Science and Engineering, Akita University, Tegata Gakuen-machi, Akita 010-8502, Japan
}

Correspondence should be addressed to Xi Liu; liuxi@mail.lzjtu.cn

Received 13 September 2014; Revised 24 November 2014; Accepted 8 December 2014

Academic Editor: Jiamin Wu

Copyright (C) 2015 Xi Liu et al. This is an open access article distributed under the Creative Commons Attribution License, which permits unrestricted use, distribution, and reproduction in any medium, provided the original work is properly cited.

\begin{abstract}
$\mathrm{FeCo} / \mathrm{Ru} / \mathrm{FeCo}$ exchange coupled synthetic antiferromagnetic multilayers were prepared with two sputtering modes. One is continuous sputtering mode, and the other mode is layer-by-layer sputtering mode. The former mode implies that substrate faced the target and film growth process was continuous when FeCo layers were sputtering, whereas the latter implies that substrate was rotating with the mask at a speed of $5 \mathrm{rpm}$ when FeCo layers were sputtering. It was found that the exchange coupling field $H_{\text {ex }}$ of sample sputtered by layer-by-layer mode was higher than the one sputtered by continuous mode. Domain structures were measured with applying varied in-plane magnetic fields along the easy axis in order to study the magnetization reversal process. We found it is a domain wall move process. When the applied field is smaller than $H_{e x}$, both the two magnetic layers have domain structure and the domain structure of the two layers is reversed correspondingly. When the applied field is varying in the range of $-H_{\mathrm{ex}}$ to $H_{\mathrm{ex}}$, the domain wall of the two layers moves correspondingly at the same time.
\end{abstract}

\section{Introduction}

Synthetic antiferromagnetic (SAF) multilayer [1] is comprised of two ferromagnetic layers separated by a nonmagnetic metallic spacer layer in which the ferromagnetic layers are coupled through antiferromagnetic interlayer exchange. Such interlayer coupling is subject to the thickness of the nonmagnetic layer [2] and is highly sensitive to structural defects [3]. In an ideal situation with proper thickness of nonmagnetic layer and low structural defects, no remanence can be observed in easy axis and the total magnetization becomes zero when the applied magnetic field is smaller than exchange coupling field $H_{\mathrm{ex}}$. In recent years, there have been many studies on SAF multilayers, as it shows great promise as soft underlayers for perpendicular recording media $[4,5]$, high moment write head material [6], and high frequency application [7]. But the reversal process in $\mathrm{FeCo} / \mathrm{Ru} / \mathrm{FeCo}$ SAF multilayers has not been studied yet. It is not known if the reversal process is a domain wall move process or moment coherent rotation process.
In this present study, $\mathrm{FeCo} / \mathrm{Ru} / \mathrm{FeCo} \mathrm{SAF}$ layers were prepared under two different FeCo sputtering modes which significantly changed the surface intermixing, so different exchange coupling fields $H_{\text {ex }}$ were measured. MFM measurements with different applying in-plane magnetic fields in the easy axis were done to study the reversal process in $\mathrm{FeCo} / \mathrm{Ru} / \mathrm{FeCo}$ multilayers.

\section{Experiment}

SAF multilayers $\mathrm{Ru} / \mathrm{FeCo}(10 \mathrm{~nm}) / \mathrm{Ru}\left(d_{\mathrm{Ru}} \mathrm{nm}\right) / \mathrm{FeCo}(10 \mathrm{~nm}) /$ $\mathrm{Ru}$ were prepared on $\mathrm{SiO}_{2}$ substrate by using DC magnetron sputtering with a $\mathrm{Fe}_{70} \mathrm{Co}_{30}$ target and a $\mathrm{Ru}$ target. The base pressure was lower than $3 \times 10^{-5} \mathrm{~Pa}$. A magnetic field around 100 Oe was applied along the substrate surface during deposition for inducing in-plane magnetic anisotropy in the films. Ru layers are deposited with power of $15 \mathrm{~W}$, Ar-gas pressure of $0.5 \mathrm{~Pa}$, and substrate rotating speed of $5 \mathrm{rpm}$. The thickness of $\mathrm{Ru}$ interlayer $d_{\mathrm{Ru}}$ varied from $0.60 \mathrm{~nm}$ to $1.20 \mathrm{~nm}$. All samples used a Ru seed layer $(2 \mathrm{~nm})$ and a $\mathrm{Ru}$ 


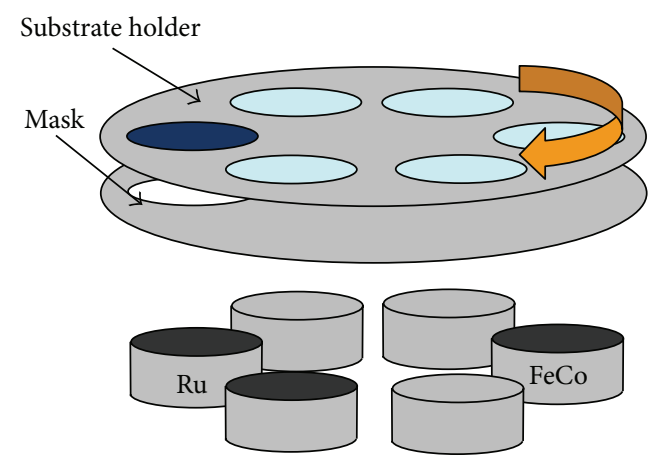

FIGURE 1: Schematic diagram of sputter system.

TABlE 1: Deposition conditions of two series samples.

\begin{tabular}{lcccc}
\hline & Power & Ar pressure & Rotating speed & Sputtering rate \\
\hline Series A & $10.0 \mathrm{~W}$ & $0.2 \mathrm{~Pa}$ & No rotation & $0.10 \mathrm{~nm} / \mathrm{sec}$ \\
Series B & $17.8 \mathrm{~W}$ & $0.2 \mathrm{~Pa}$ & $5 \mathrm{rpm}$ & $0.25 \mathrm{~nm} / \mathrm{rot}$ \\
\hline
\end{tabular}

capping layer $(3 \mathrm{~nm})$. The multilayers were prepared in a multilayer sputtering system and the schematic diagram is shown in Figure 1. We prepared two series samples, and the conditions are given in Table 1 . FeCo layer of series A was prepared with a continuous sputtering mode: substrates were not rotating and facing FeCo target through the hole in the mask during deposition. FeCo layer of series B was prepared with a layer-by-layer sputtering mode: substrates and the mask were rotating at the same speed.

Magnetic properties were measured by a vibrating sample magnetometer (VSM). The domain structure was measured by a magnetic force microscopy (MFM) applying different inplane magnetic fields along easy-axis. A FePt tip was adopted and measurement conditions were vibrating the amplitude of the tip of $15 \mathrm{~nm}$ for morphology measurement and $3 \mathrm{~nm}$ for phase measurement with scan height of about $10 \mathrm{~nm}$ and constant $Q$ factor of about 6000 . Before being measured, FePt tip was magnetized by VSM along the pinpoint direction.

\section{Results and Discussions}

Upon investigation of the relationship between the exchange coupling field $H_{\mathrm{ex}}$ and the thickness of Ru interlayer $d_{\mathrm{Ru}}$ in SAF films, it was found that the $H_{\mathrm{ex}}$ of SAF coupled structures is related to $d_{\mathrm{Ru}}$. As shown in Figure 2, with the increase of $d_{\mathrm{Ru}}$, the $H_{\mathrm{ex}}$ of series $\mathrm{B}$ samples increases and reaches a maximum value when $d_{\mathrm{Ru}}=0.90 \mathrm{~nm}$, and when $d_{\mathrm{Ru}}$ increases further $\left(d_{\mathrm{Ru}}>0.90 \mathrm{~nm}\right), H_{\mathrm{ex}}$ decreases drastically. We also found that the $H_{\mathrm{ex}}$ of series A samples had the same results: the $H_{\mathrm{ex}}$ value got maximum when $d_{\mathrm{Ru}}=0.90 \mathrm{~nm}$ but the value of series B samples was much larger than series A samples. Figures 3(a) and 3(b) show the $M-H$ of two series samples with $d_{\mathrm{Ru}}=0.90 \mathrm{~nm}$. It was shown that the exchange coupling field $H_{\text {ex }}$ of series B sample is higher than series A sample: about 185 Oe for series B sample and about 80 Oe for series A sample. Figures 3(c) and 3(d) show two series samples without $\mathrm{Ru}$ spacer layer. It was shown that the induced anisotropy field $H_{k}$ of series B sample is higher than series A sample. This result agrees with the SAF layers' result, because the effective anisotropy field $H_{k}$ relates to the exchange coupling field $H_{\mathrm{ex}}$ in SAF sandwich films [7]. It is well known that the interlayer coupling of samples grown by $\mathrm{MBE}$ is larger than that grown by sputtering, because of the larger surface intermixing in the sputtered samples due to the substrate bombardment by neutral atoms. This gives rise to random component in the atom distribution near the interface and to a destructive interference of the scattered electronic waves, so the coupling is frustrated $[8,9]$. We think the growth process of series B samples is similar to $\mathrm{MBE}$ process, and it can effectively decrease the bombardment by neutral atoms compared with the growth process of series A samples. So we can conclude that the layer-by-layer growth mode can decease the surface intermixing and get laser induced anisotropy field $H_{k}$ and exchange coupling field $H_{\mathrm{ex}}$.

In order to study the reversal process of SAF multilayers, we measured MFM images applying varied in-plane magnetic field along easy-axis. From the MFM measurement we found the surface of the two series samples is very flat and the average roughness is less than $0.1 \mathrm{~nm}$. Figure 4 shows the MFM images of series B sample with $d_{\mathrm{Ru}}=0.90 \mathrm{~nm}$ measured with different in-plane magnetic field. The magnetic field applied along the easy-axis direction and ranged from 1500 Oe to -1500 Oe. Because of the multilayer structure, we can just measure domain structure of upper FeCo layer. But we can deduce the bottom layer's domain structure according to magnetic properties of exchange coupled SAF multilayers. Figure 4(a) shows MFM images measured when the asdeposited sample had not been applied magnetic field, we can see clear domain structure. Figure 4(b) shows MFM images measured with 1500 Oe in-plane magnetic field, and we cannot see very clear domain wall structure, which means magnetization is saturated and almost all magnetic moments array along the applied field. From Figure 4(c) it can be seen when applied magnetic field decreases to 1000 Oe, many small reverse domains appear. Figure 4(f) is measured when the applied in-plane magnetic field decreases from $1500 \mathrm{Oe}$ to 200 Oe. From Figures 4(c)-4(f), it can be seen those small reversed magnetic domains grow bigger and the domain wall moves with change of the applied in-plane magnetic field. Figures $4(\mathrm{~g})-4(\mathrm{l})$ were measured with the applied inplane magnetic field range from 150 Oe to -200 Oe. From $M-H$ loop we can get the total magnetization of film are zero when the applied magnetic field are smaller than the exchange coupling field $H_{\mathrm{ex}}$. But we can see clearly domain wall structure, so we can deduce that the bottom FeCo layer has the same domain structure as the upper layer, but the magnetization direction is reverse correspondingly. From marked parts in Figures 4(g)-4(l) we can see the domain wall moves process, so we can deduce that the domain wall of the two layers moves correspondingly at the same time. Figures $4(\mathrm{~m})-4(\mathrm{p})$ show the MFM images measured when the applied magnetic field ranges from -300 Oe to -1500 Oe, we can see the positive domain becomes smaller and disappears. So, we can conclude that the reversal process in SAF multilayers is a domain wall move process, and the domain wall in both upper layer and bottom layer moves 


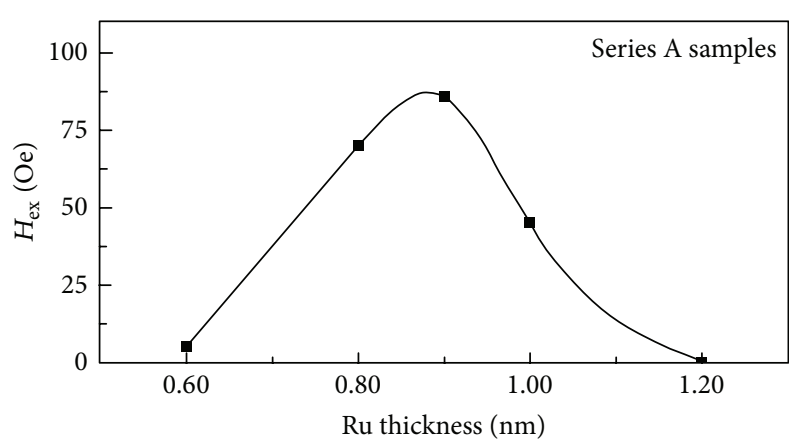

(a)

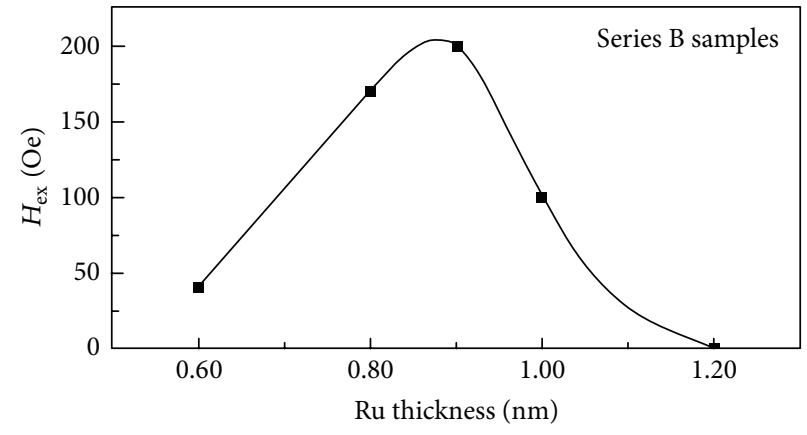

(b)

Figure 2: Dependence of $H_{\mathrm{ex}}$ of series A and B samples on $d_{\mathrm{Ru}}$.

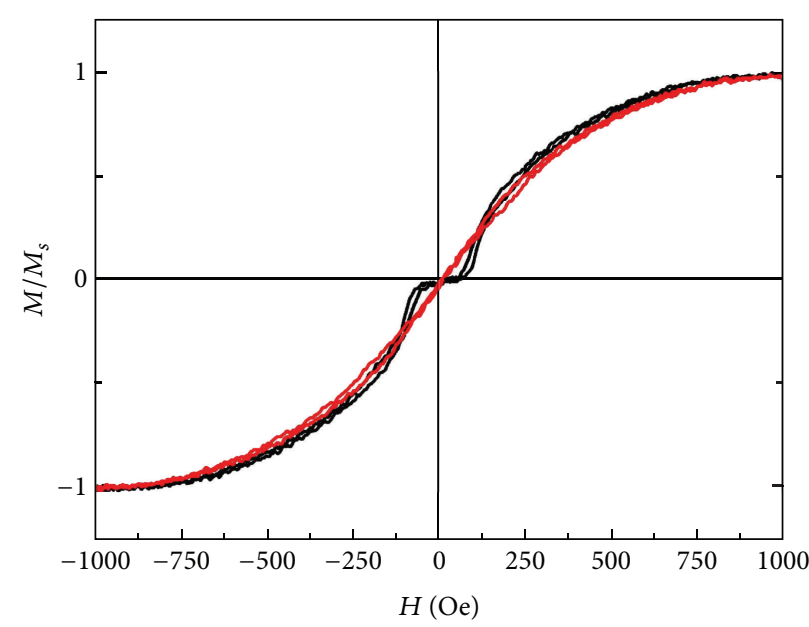

(a)

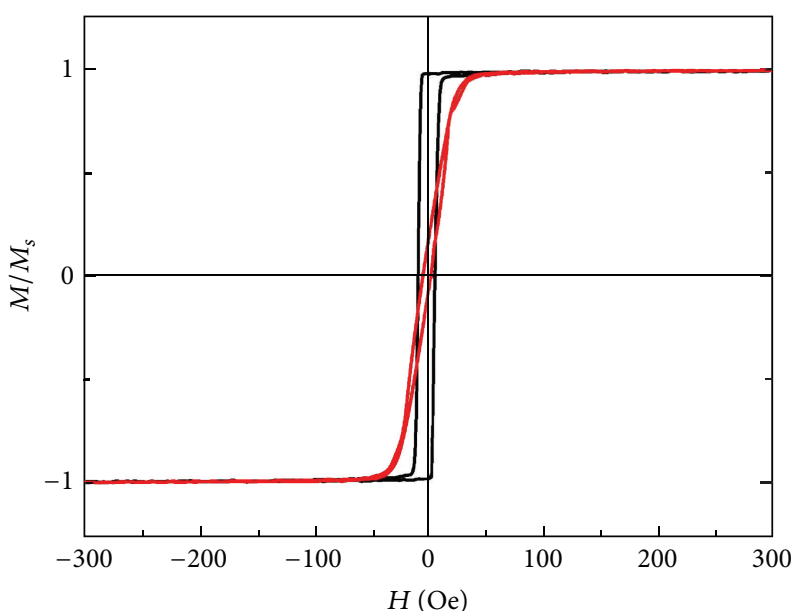

— Easy axis — Hard axis

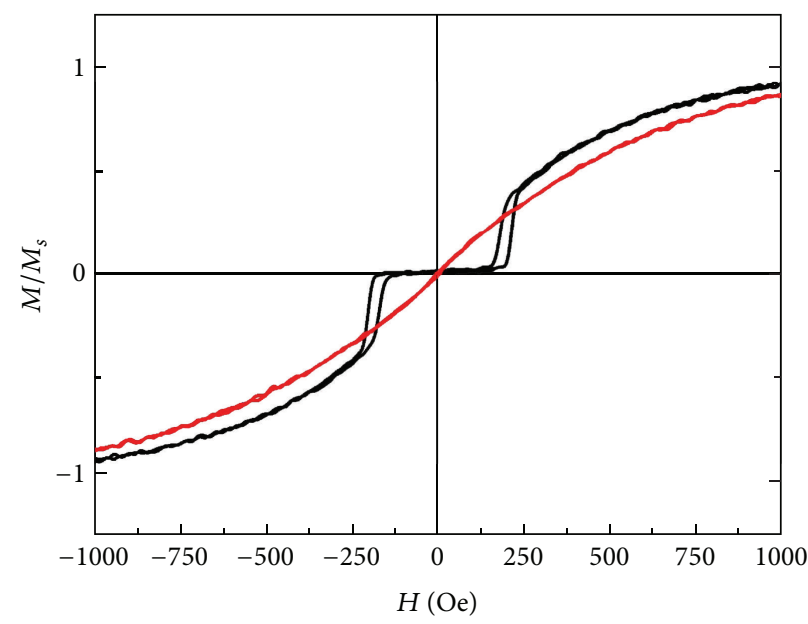

(b)

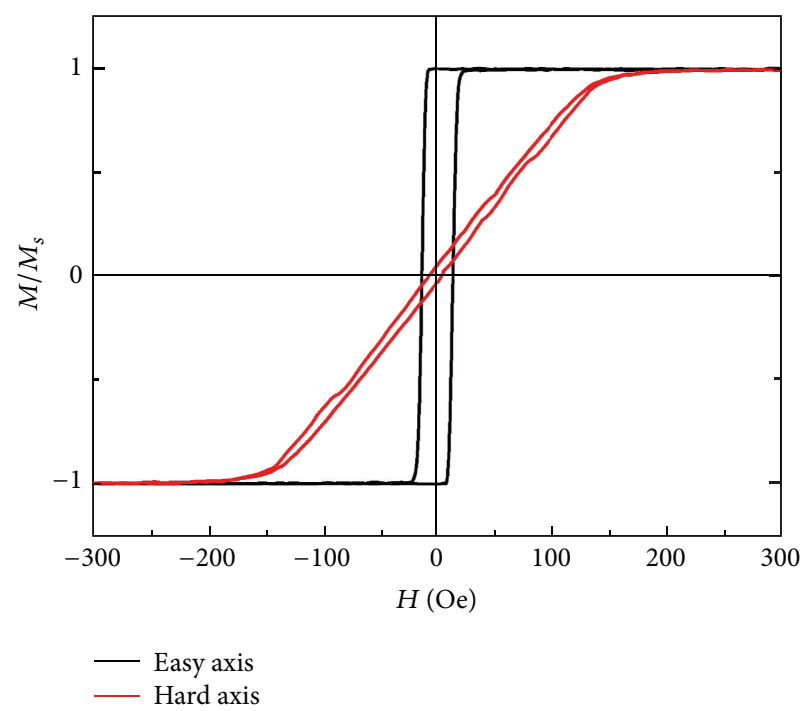

(d)

FIGURE 3: $M-H$ loop of (a) series A sample with $d_{\mathrm{Ru}}=0.90 \mathrm{~nm}$, (b) series B sample with $d_{\mathrm{Ru}}=0.90 \mathrm{~nm}$, (c) series A sample without Ru spacer layer, (d) series B sample without Ru spacer layer. 


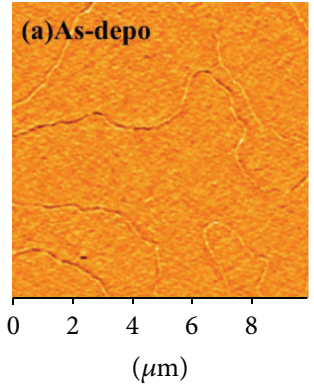

(a)

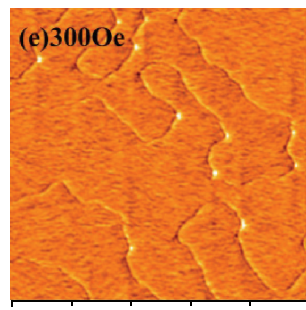

$\begin{array}{lllll}0 & 2 & 4 & 6 & 8\end{array}$

$(\mu \mathrm{m})$

(e)

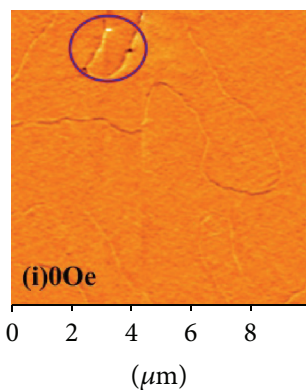

(i)

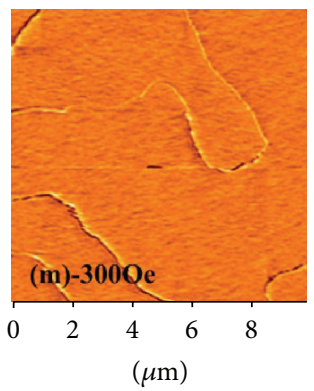

(m)

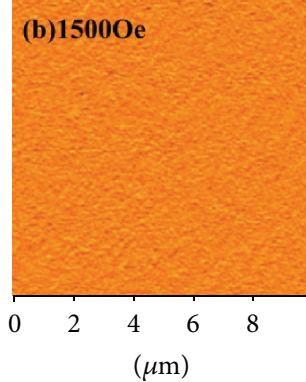

(b)

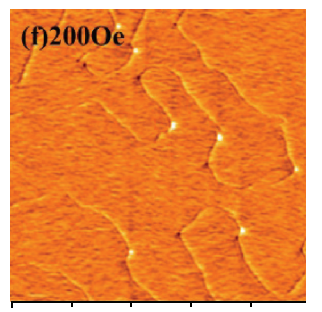

(f)

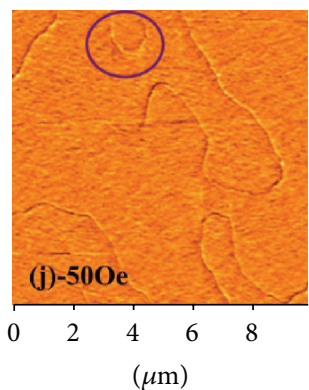

(j)

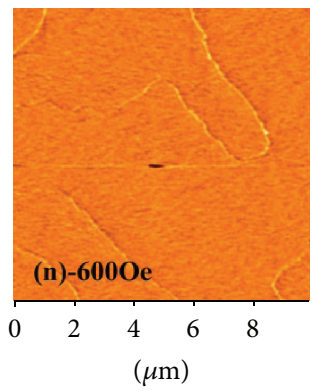

(n)

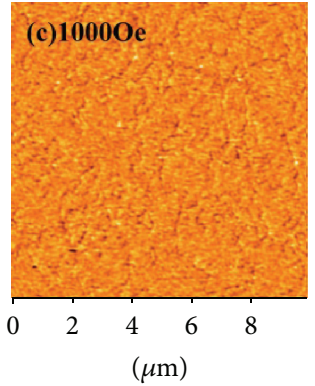

(c)

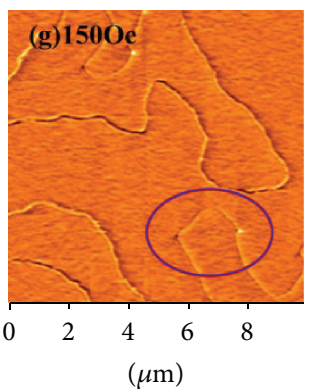

(g)

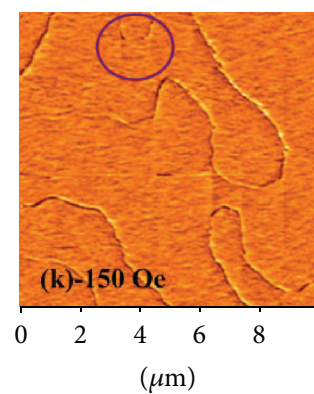

(k)

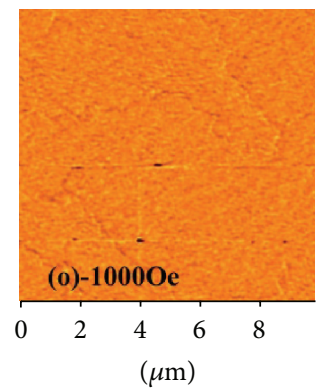

(o)

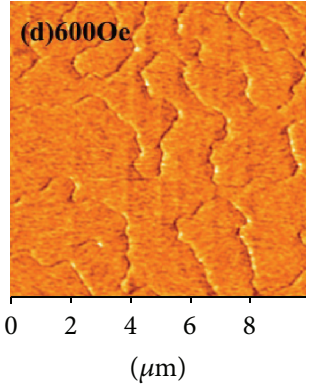

(d)

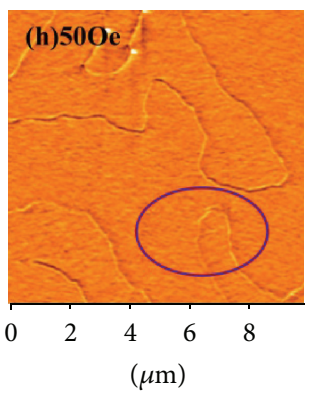

(h)

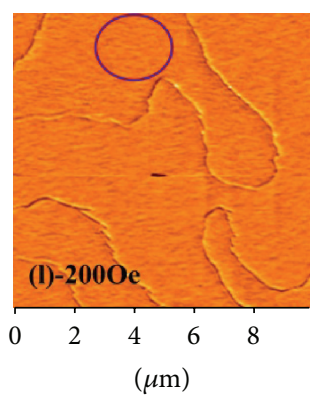

(1)

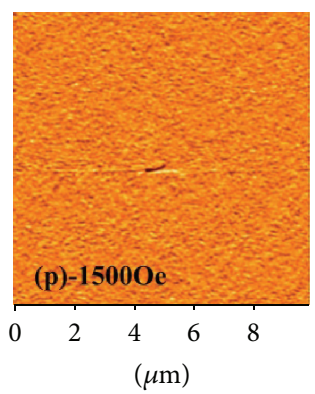

(p)

Figure 4: MFM images of $\mathrm{FeCo}(10 \mathrm{~nm}) / \mathrm{Ru}(0.90 \mathrm{~nm}) / \mathrm{FeCo}(10 \mathrm{~nm})$ prepared with layer-by-layer mode measured with in-plane magnetic field range from 1500 Oe to -1500 Oe.

with the varying in-plane magnetic fields; when the in-plane magnetic field is smaller than exchange coupling field $H_{\mathrm{ex}}$, the domain structure in the two layers is the same but the magnetization direction is reverse correspondingly, and the domain wall in the two layers moves correspondingly at the same time.

\section{Conclusion}

Synthetic antiferromagnetic multilayers $\mathrm{Ru} / \mathrm{FeCo}(10 \mathrm{~nm}) /$ $\mathrm{Ru}\left(d_{\mathrm{Ru}} \mathrm{nm}\right) / \mathrm{FeCo}(10 \mathrm{~nm}) / \mathrm{Ru}$ were prepared with two FeCo sputtering mode: continuous mode and layer-by-layer mode. The exchange coupling field $H_{\text {ex }}$ of SAF film deposited by 
layer-by-layer mode is higher than samples deposited by continuous mode because of decrease of surface intermixing due to the substrate bombardment by neutral atoms. The magnetization reversal process is a domain wall move process. When the applied field was smaller than $H_{\text {ex }}$, both the two magnetic layers have same domain structure and the domain structure of the two layers was reverse correspondingly. It is also found that the domain wall of the two magnetic layers move correspondingly at the same time when the applied field is smaller than $H_{\mathrm{ex}}$ and changing.

\section{Conflict of Interests}

The authors declare that there is no conflict of interests regarding the publication of this paper.

\section{Acknowledgment}

This study was supported by the Science Foundation for Young Scholars of Lanzhou Jiaotong University (Grant no. 2013036).

\section{References}

[1] P. Grünberg, R. Schreiber, Y. Pang, U. Walz, M. B. Brodsky, and H. Sowers, "Layered magnetic structures: evidence for antiferromagnetic coupling of Fe layers across Cr interlayers," Physical Review Letters, vol. 57, no. 19, pp. 2442-2445, 1986.

[2] S. S. P. Parkin, N. More, and K. P. Roche, "Oscillations in exchange coupling and magnetoresistance in metallic superlattice structures: $\mathrm{Co} / \mathrm{Ru}, \mathrm{Co} / \mathrm{Cr}$, and Fe/Cr," Physical Review Letters, vol. 64, no. 19, pp. 2304-2307, 1990.

[3] C. H. Marrows, B. J. Hickey, M. Herrmann et al., "Damage caused to interlayer coupling of magnetic multilayers by residual gases," Physical Review B-Condensed Matter and Materials Physics, vol. 61, article 4131, 2000.

[4] Y. Kawato, M. Futamo, and K. Nakamoto, "Perpendicular magnetic recording medium and magnetic storage apparatus," U.S. Patent no. 20020028356 A1, (2002).

[5] S. C. Byeon, A. Misra, and W. D. Doyle, "Synthetic antiferromagnetic soft underlayers for perpendicular recording media," IEEE Transactions on Magnetics, vol. 40, no. 4, pp. 2386-2388, 2004.

[6] Y. Chen, K. Sin, H. Jiang et al., "High moment materials and fabrication processes for shielded perpendicular write head beyond 200 Gb/in2," IEEE Transactions on Magnetics, vol. 43, no. 2, pp. 609-614, 2007.

[7] L. Zhang, W. Zhang, X. Liu et al., "High frequency characteristics of synthetic antiferromagnetic coupling FeCoN sandwich films," IEEE Transactions on Magnetics, vol. 47, no. 10, pp. 39323934, 2011.

[8] P. Bruno and C. Chappert, "Ruderman-Kittel theory of oscillatory interlayer exchange coupling," Physical Review B, vol. 46, no. 1, pp. 261-270, 1992.

[9] Y. Wang, P. M. Levy, and J. L. Fry, "Interlayer magnetic coupling in Fe/Cr multilayered structures," Physical Review Letters, vol. 65, no. 21, pp. 2732-2735, 1990. 

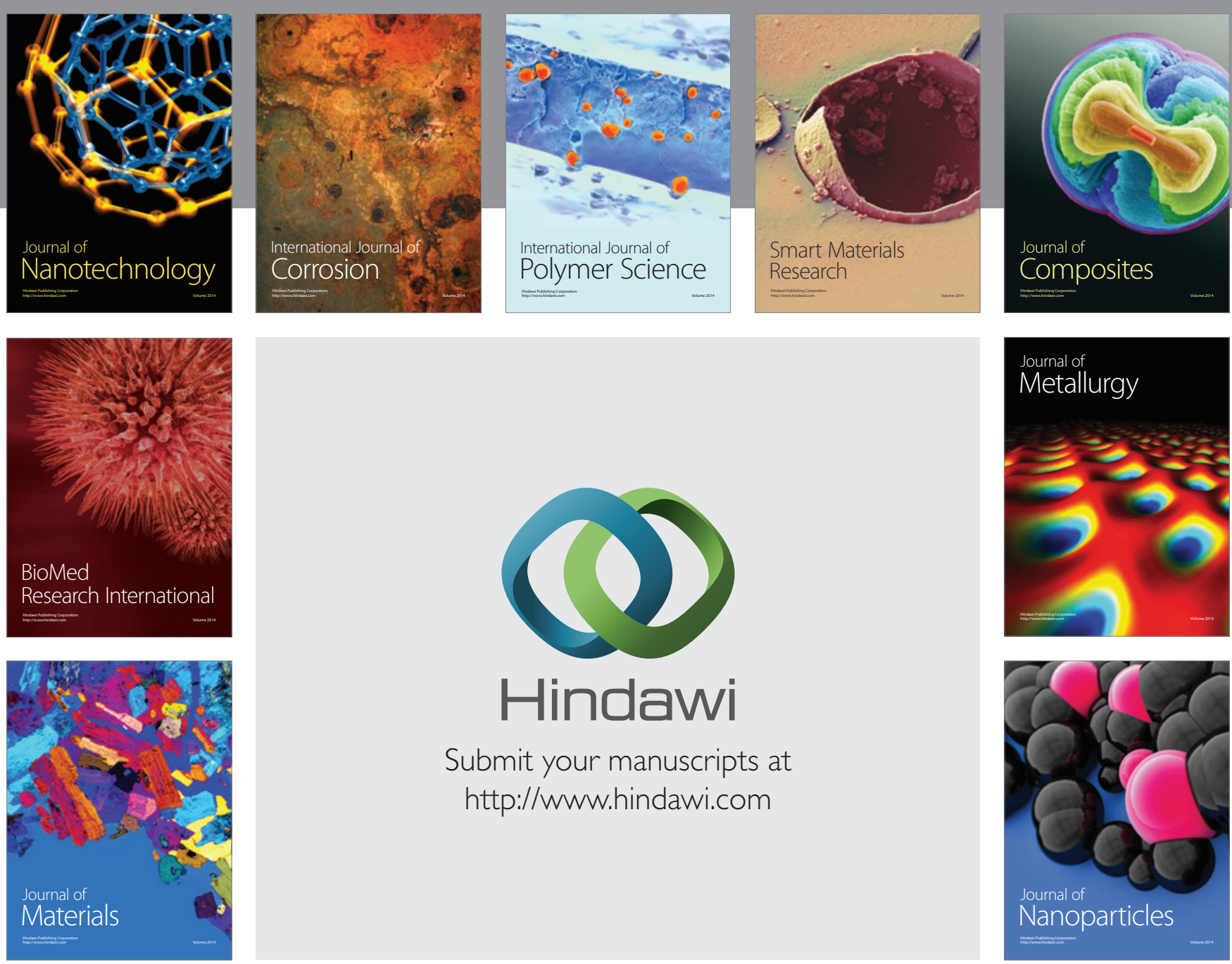

Submit your manuscripts at http://www.hindawi.com
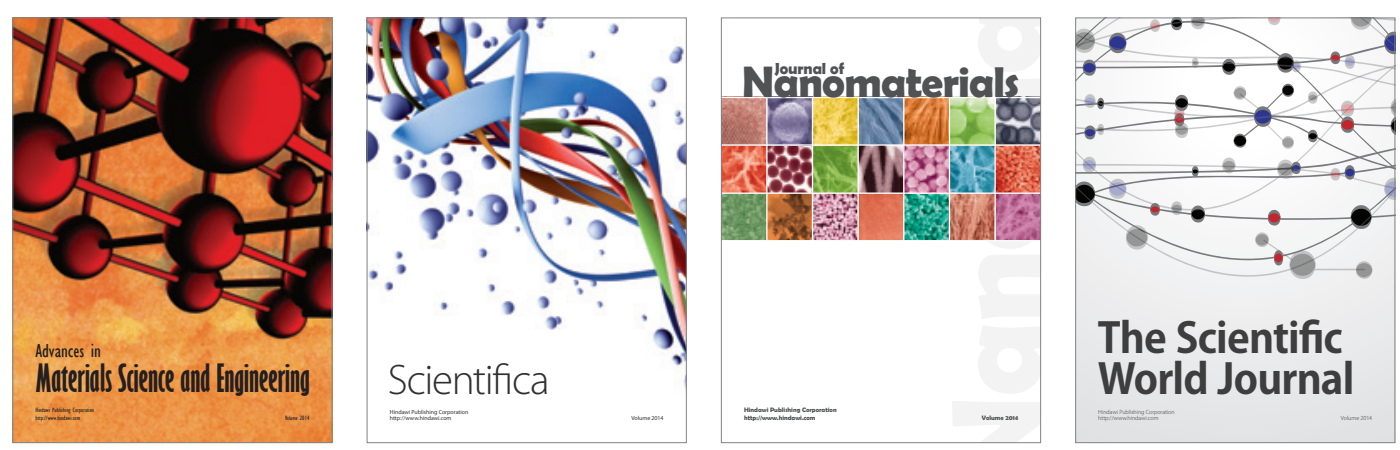

\section{The Scientific World Journal}
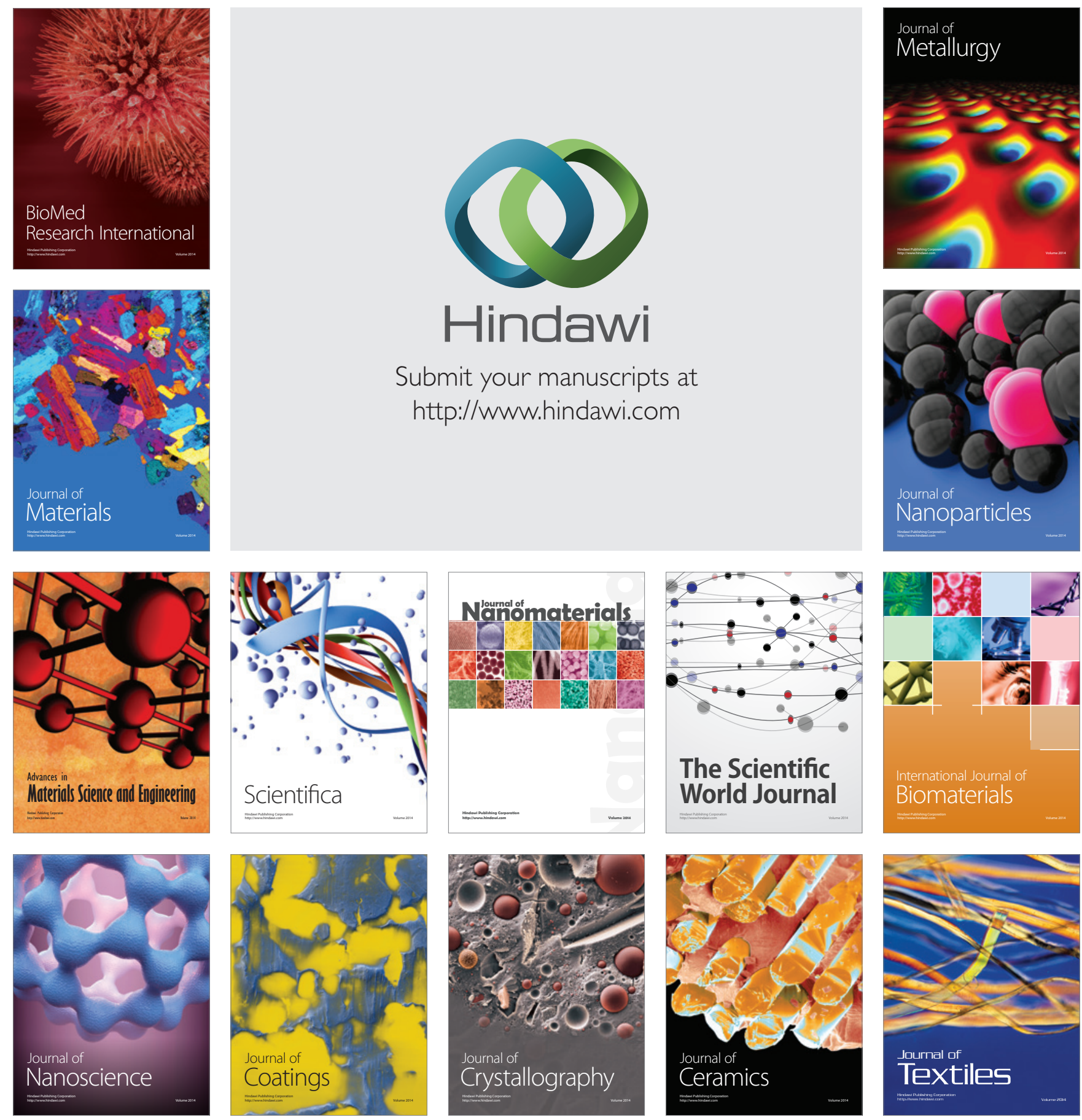